\title{
Saúde coletiva e saúde pública no currículo dos cursos de educação física: uma revisão sistemática
}

\author{
Collective health and public health in physical education \\ courses curriculum: a systematic review
}

\section{Salud colectiva y salud pública en el currículo de cursos de educación física: una revisión sistemática}

\author{
(iD) Vítor Tavares da Silva \\ Universidade Federal de Pelotas, Pelotas, Rio Grande do Sul, Brasil \\ vitortavarees@outlook.com \\ iD Cintia Ramos Nicoes \\ Universidade Federal de Pelotas, Pelotas, Rio Grande do Sul, Brasil \\ cintianicoes@hotmail.com \\ iD) Alan Goularte Knuth \\ Universidade Federal do Rio Grande, Rio Grande, Rio Grande do Sul, Brasil \\ Alan_knuth@yahoo.com.br
}

\begin{abstract}
Resumo: Este estudo objetivou revisar as publicações que tematizam a Saúde Coletiva (SC) e a Saúde Pública (SP) no currículo dos cursos superiores de Educação Física (EF) no Brasil. Foi conduzida uma revisão sistemática da literatura. As buscas foram realizadas nas bases de dados LILACS, PubMed, SciELO e SPORTDiscus por meio da combinação dos descritores e termos "saúde pública" OR "saúde coletiva" AND “currículo" AND "educação física". Seis pesquisas foram incluídas. Os artigos indicam pouca incorporação das temáticas de SC e SP pelos cursos de formação em EF. Ademais, aparecem majoritariamente na formação de cursos de bacharelado. Outrossim, mesmo presentes nos currículos, figuram de
\end{abstract}


maneira frágil, com insuficiente densidade para as interlocuções com os serviços públicos de saúde.

Palavras-chave: Educação física. Currículo. Saúde pública. Saúde coletiva.

Abstract: This study aimed to review the publications that focus on Collective Health $(\mathrm{CH})$ and Public Health $(\mathrm{PH})$ in the curriculum of Physical Education courses (PE) in Brazil. A systematic literature review was conducted. The searches were performed in the LILACS, PubMed, SciELO and SPORTDiscus databases, by combining the descriptors and terms "collective health" OR "public health" AND "curriculum" AND "physical education". Six researshes were included. The articles indicate little incorporation of $\mathrm{CH}$ and $\mathrm{PH}$ themes into PE training courses. In addition, they appear mostly in the formation of baccalaureate courses. Moreover, even thought they are present in the curriculum, they appear inconsistent and without sufficient density for interlocutions with public health services.

Keywords: physical education. curriculum. public health. collective health.

Resumen: Este estudio tuvo como objetivo revisar las publicaciones que se centran en Salud Colectiva (SC) y Salud Pública (SP) en currículo de los cursos de Educación Física (EF) en Brasil. Se realizó una revisión sistemática de la literatura. Las búsquedas se realizaron en las bases de datos LILACS, PubMed, SciELO y SPORTDiscus, combinando los descriptores y los términos "saúde pública" OR "saúde coletiva" AND "currículo" AND "educação física". Se incluyeron seis encuestas. Los artículos indican poca incorporación de temas SC y SP en los cursos de capacitación en EF. Además, aparecen principalmente en la formación de cursos de bachillerato. Además, incluso presentes en los currículos, parecen frágiles con densidad insuficiente para las interlocuciones con los servicios de salud pública.

Palabras clave: educación física. curriculum. salud pública. salud colectiva.

Submetido em: 03-11-2019

Aceito em: 08-05-2020 
Saúde coletiva e saúde pública no currículo dos cursos de educação física... Vítor Tavares da Silva • Cintia Ramos Nicoes • Alan Goularte Knuth

\section{Introdução}

Mudanças concretas em torno da formação dos profissionais do campo da saúde costumam evoluir de forma lenta e exigem constante interesse acadêmico, principalmente quando focado na atuação profissional voltada para a esfera da saúde pública (SP) e no sentido de sua relevância social. Apesar de o núcleo da Educação Física (EF), nos últimos anos, figurar junto às principais políticas de SP por intermédio do eixo atividade física e práticas corporais, sabe-se que, historicamente, o curso de EF não possui uma formação inicial alinhada para esse campo de intervenção profissional no Brasil (CARVALHO, NOGUEIRA, 2016).

Em nível de graduação, além dos dilemas das diferentes modalidades de formação (bacharelado e licenciatura), os processos formativos da EF estiveram, hegemonicamente, distantes dos debates de um pensamento crítico sobre a formação profissional em saúde, mantendo características ainda conservadoras em relação a discussões e práticas biologizadas, fragmentadas, centradas na instrução técnica e mecanicista, porém alguns avanços foram experimentados nos últimos anos (FREITAS; BRASIL; SILVA, 2006; CARVALHO, 2007).

Dessa maneira, alicerçadas na Saúde Coletiva (SC) e seus três pilares de conhecimento - a epidemiologia, as ciências sociais e humanas e o planejamento, gestão e políticas em saúde -, as ciências da saúde, as quais contam com a EF, aproximam-se de uma proposta mais afinada com uma prática cuidadora, pensada para a atenção integral de indivíduos e coletivos, a qual extrapola, mas não exclui a dimensão biológica do corpo. Essa perspectiva destaca uma ideia que amplia o conceito de saúde, contemplando aspectos sociais, subjetivos, históricos, afetivos e culturais (CARVALHO; CECCIM, 2006).

Nesse prisma, um dos alvos de estudo e intervenção do campo de conhecimentos da SC é a SP, a qual é entendida como o conjunto de medidas executadas pelo Estado, e, por vezes, reunindo 
Saúde coletiva e saúde pública no currículo dos cursos de educação física...

Vítor Tavares da Silva • Cintia Ramos Nicoes • Alan Goularte Knuth

prestadores e compradores de serviço da esfera pública e privada, buscando eficiência nos serviços e a saúde da população, ainda que ocorram distorções e interesses corporativos, compondo uma rede complexa (PAIM, 2018).

Nesse sentido, uma das formas de nos aproximarmos da compreensão das práticas experimentadas nos processos formativos da EF em relação à SC e à SP se manifesta junto à estruturação em que são sistematizadas as ações e intenções sobre os conteúdos a serem desenvolvidos nos cursos de formação: o currículo. Uma proposta curricular define qual é a trajetória a ser seguida na programação dos conteúdos que um curso abrange, evidenciando as concepções das instituições de ensino e identificando quais as finalidades e metas da formação oferecida (COLL, 2002).

Entretanto, houve muitos avanços na área dos estudos do currículo, que ampliam essa discussão, superando as teorias tradicionais de maneira a alcançar questões mais amplas. Dessa forma, emergem os conceitos de ideologia e poder, considerando aportes curriculares sociológicos e filosóficos, enquanto entende-se o currículo como algo dialético e dinâmico, avançando até aspectos de identidade, alteridade, diferença, subjetividade e representação, quando se tem um debate mais reflexivo sobre a atual sociedade (SILVA, 2005).

Com isso, na ênfase da temática da SC e da SP na formação de Profissionais de Educação Física (PEF), ainda não se encontram consensos, contando com investigações pontuais e dissipadas dentro do cenário dos cursos superiores em EF brasileiros. Por vezes também, a SC e a SP apresentam-se como sinônimos ou com divergências conceituais. Dessa forma, a literatura científica não dispõe de uma análise criteriosa de publicações que sintetize o cenário de resultados e discussões ligadas a SC e a SP nos currículos dos cursos superiores de EF no Brasil nos últimos 15 anos, em que se tem uma formação dividida entre bacharelado e licenciatura, quando se intensificaram as interações entre EF, SC e SP. 
Saúde coletiva e saúde pública no currículo dos cursos de educação física...

Vítor Tavares da Silva • Cintia Ramos Nicoes • Alan Goularte Knuth

Portanto, o objetivo deste estudo foi revisar as publicações que tematizam a SC e a SP nos currículos dos cursos superiores de EF no Brasil.

\section{Métodos}

Este estudo se caracteriza como uma revisão sistemática da literatura a partir das recomendações do Preferred Reporting Items for Systematic reviews and Meta-Analyses (PRISMA). O projeto de pesquisa conta com registro na base eletrônica International Prospective Register of Systematic Reviews (PROSPERO), sob ID 122385. As buscas foram realizadas nas bases de dados LILACS, PubMed, SciELO e SPORTDiscus por um dos pesquisadores. O processo de triagem contou com descritores e termos baseados nas sugestões dos Descritores em Ciências da Saúde (DeCS) e do Medical Subject Headings (MeSH), empregados de acordo com as combinações "saúde pública" OR "saúde coletiva" AND "currículo" $A N D$ "educação física" em português, bem como "public health" $O R$ "collective health" AND "curriculum" AND "physical education" em inglês.

As buscas se deram no dia 18 de dezembro de 2018. Foram adotados os seguintes critérios de inclusão para seleção: artigos de natureza científica, publicados na língua inglesa e portuguesa, em que os descritores e termos utilizados deveriam constar no título ou resumo de manuscritos do tipo artigo original, disponíveis na íntegra, que versassem sobre pesquisas no Brasil, publicados entre 2004 e 2018. O critério temporal foi adotado em função da instituição da reforma curricular de 2004 (Resolução CNE/CES n.07/2004), que, por meio das Diretrizes Curriculares Nacionais (DCN), estabeleceu mudanças para os cursos de graduação em EF (BRASIL, 2004).

Como critérios de exclusão foram considerados: duplicidade nas bases de dados, produções do tipo ensaio, tese, dissertação e carta editorial, bem como estudos que dedicavam-se a currículos 
Saúde coletiva e saúde pública no currículo dos cursos de educação física...

Vítor Tavares da Silva • Cintia Ramos Nicoes • Alan Goularte Knuth

concebidos antes das DCN de 2004. As referências bibliográficas dos estudos localizados nas bases de dados também foram revisadas para localizar outras pesquisas de potencial interesse ao tema, sob os mesmos critérios de seus artigos de origem. Com isso, dois pesquisadores, de forma independente, procederam com as etapas de análise de títulos e análise de resumos.

Após a seleção inicial, realizou-se a análise de todas as publicações na íntegra, quando, de forma consensual, foram determinados os estudos a serem incluídos e as listas de referências analisadas. Em caso de divergência entre a inclusão ou exclusão de algum artigo, um terceiro pesquisador executara as etapas de análise supracitadas.

Os dados de cada um dos estudos foram tabulados em planiIha do software Microsoft Excel ${ }^{\circledR}$ (pacote Microsoft Office ${ }^{\circledR}$ versão 2013, desenvolvido pela Microsoft $\circledast$ ) com informações sobre os estudos incluídos e seus principais resultados.

\section{Resultados e discussão}

Após as buscas, foram encontrados 194 estudos, sendo selecionados seis artigos originais que elucidavam as temáticas da SP e SC nos currículos dos cursos superiores de EF no Brasil segundo os critérios desta revisão (Figura 1). As pesquisas foram localizadas em revistas científicas nacionais, bem como estão disponíveis em português e possuem livre acesso. Os artigos selecionados e suas principais características são descritas no Quadro 1. 
Saúde coletiva e saúde pública no currículo dos cursos de educação física...

Vítor Tavares da Silva • Cintia Ramos Nicoes • Alan Goularte Knuth

Figura 1 - Fluxograma: processo de seleção dos estudos. Saúde Coletiva e Saúde Pública nos currículos da Educação Física, Brasil 2019

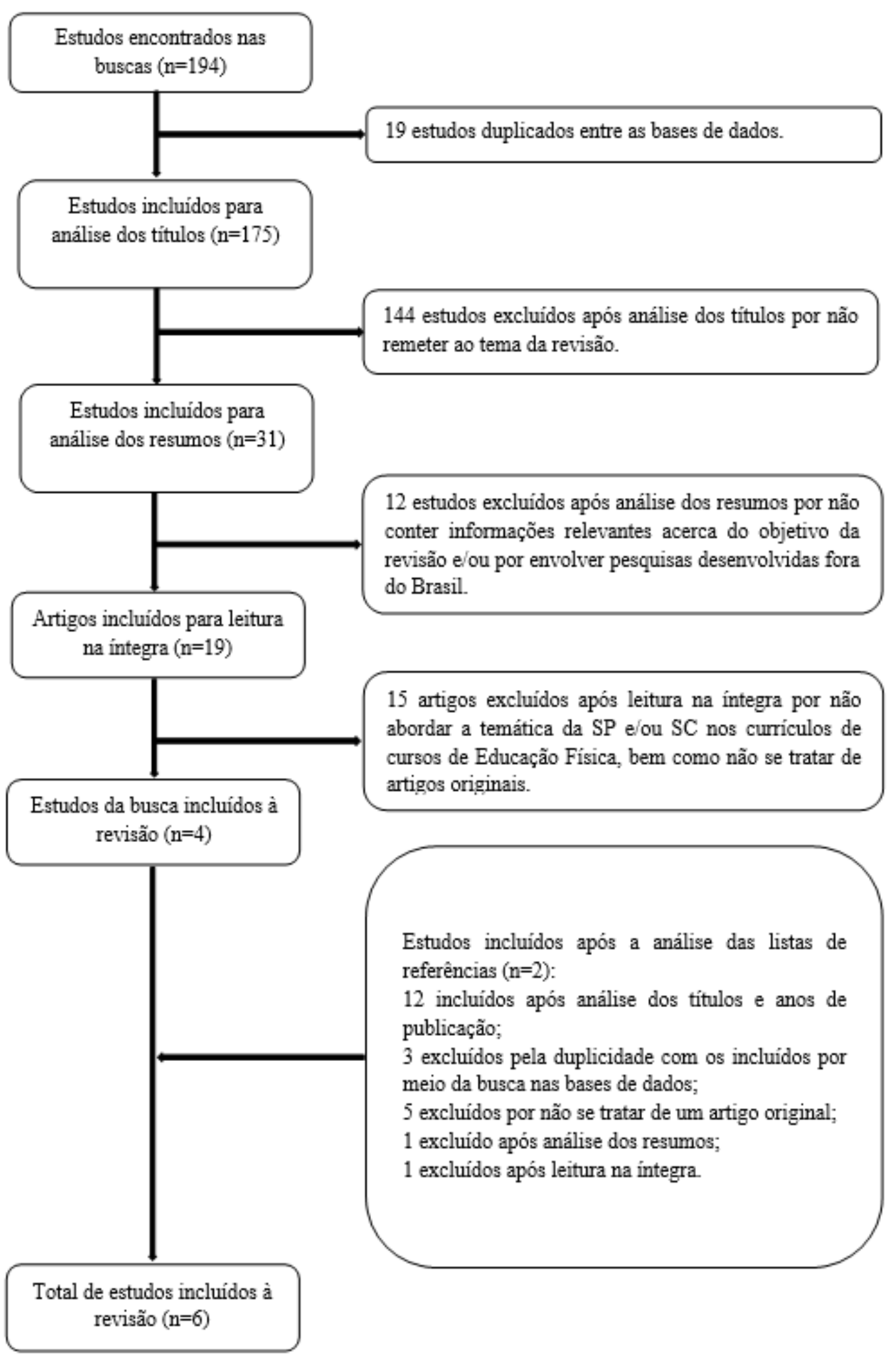


Saúde coletiva e saúde pública no currículo dos cursos de educação física...

Vítor Tavares da Silva • Cintia Ramos Nicoes • Alan Goularte Knuth

\section{Quadro 1 - Detalhamento dos estudos. Saúde Coletiva e Saúde Pública nos currículos da Educação Física, Brasil 2019}

\begin{tabular}{|c|c|c|c|}
\hline Estudo & Objetivo e Método & Resultados & UF / IES \\
\hline $\begin{array}{l}\text { Anjos e } \\
\text { Duarte } \\
\text { (2009) }\end{array}$ & $\begin{array}{l}\text { Analisar currículos de facul- } \\
\text { dades de graduação em EF } \\
\text { Método: Análise Documen- } \\
\text { tal }\end{array}$ & $\begin{array}{l}\text { Não há direcionamento dos cursos } \\
\text { para o campo da saúde e os bacha- } \\
\text { réis têm mais respaldo em saúde } \\
\text { do que os licenciados; predomi- } \\
\text { nam as disciplinas de abordagem } \\
\text { curativa, prescritiva; nenhuma das } \\
\text { instituições possui o conjunto de } \\
\text { disciplinas SC, SP e algumas não as } \\
\text { contemplam; o estágio no serviço } \\
\text { público de saúde, na Atenção Bá- } \\
\text { sica, não é previsto em nenhuma } \\
\text { instituição. }\end{array}$ & $\begin{array}{c}\text { São Paulo / } 4 \\
\text { públicas e } 1 \\
\text { privada }\end{array}$ \\
\hline $\begin{array}{l}\text { Brugnerotto } \\
\text { e Simões } \\
\text { (2009) }\end{array}$ & $\begin{array}{l}\text { Analisar o conceito de saú- } \\
\text { de presente nos currículos } \\
\text { de formação em EF nas } \\
\text { principais universidades do } \\
\text { Estado do Paraná } \\
\text { Método: Análise Documen- } \\
\text { tal }\end{array}$ & $\begin{array}{l}\text { Foram levadas a cabo as recomen- } \\
\text { dações das Diretrizes Curriculares } \\
\text { específicas e por isso o conceito de } \\
\text { saúde recomendado é o da OMS, } \\
\text { direcionado à estratégia da Promo- } \\
\text { ção da Saúde. } \\
\text { Três cursos optaram pelo enfoque } \\
\text { de saúde humanista para a licen- } \\
\text { ciatura e o biológico para o bacha- } \\
\text { relado. } \\
\text { Outros três cursos optaram pela } \\
\text { inserção da disciplina apenas nos } \\
\text { cursos de bacharelado, sendo que } \\
\text { em dois o enfoque é biológico e, } \\
\text { em um, humanizado. }\end{array}$ & $\begin{array}{l}\text { Paraná / } 6 \\
\text { públicas }\end{array}$ \\
\hline $\begin{array}{c}\text { Costa et al. } \\
(2012)\end{array}$ & $\begin{array}{l}\text { Investigar a formação pro- } \\
\text { fissional em SP e SC dos } \\
\text { PEF } \\
\text { Método: Análise Documen- } \\
\text { tal }\end{array}$ & $\begin{array}{l}\text { Verificou-se, na maioria dos cursos } \\
\text { de graduação em EF, a ausência de } \\
\text { disciplinas envolvendo conteúdos } \\
\text { de SP e SC. }\end{array}$ & $\begin{array}{l}\text { Contexto na- } \\
\text { cional }\end{array}$ \\
\hline $\begin{array}{l}\text { Falci e Beli- } \\
\text { sario (2013) }\end{array}$ & $\begin{array}{l}\text { Analisar a formação do PEF } \\
\text { para sua inserção na APS, } \\
\text { em Minas Gerais, a partir } \\
\text { da visão de diferentes ato- } \\
\text { res envolvidos no proces- } \\
\text { so. } \\
\text { Método: Estudo de Caso } \\
\text { descritivo-exploratório }\end{array}$ & $\begin{array}{l}\text { Os resultados apontaram que, den- } \\
\text { tre as principais dificuldades da in- } \\
\text { serção de PEF na APS, a graduação } \\
\text { foi mencionada como insuficiente } \\
\text { para atuar na atenção primária. } \\
\text { A pós-graduação foi identificada } \\
\text { como uma das estratégias para mi- } \\
\text { nimizar essa insuficiência. } \\
\text { A superação das limitações da for- } \\
\text { mação inicial em SP e SC necessita } \\
\text { de uma reestruturação curricular. }\end{array}$ & $\begin{array}{c}\text { Minas Gerais / } \\
\text { UFMG }\end{array}$ \\
\hline
\end{tabular}


Saúde coletiva e saúde pública no currículo dos cursos de educação física... Vítor Tavares da Silva • Cintia Ramos Nicoes • Alan Goularte Knuth

\begin{tabular}{|c|c|c|c|}
\hline $\begin{array}{l}\text { Pasquim } \\
\text { (2010) }\end{array}$ & $\begin{array}{l}\text { Compreender como está } \\
\text { sendo realizado o ensino } \\
\text { de SC nos cursos de gra- } \\
\text { duação de EF em duas im- } \\
\text { portantes instituições pú- } \\
\text { blicas de ensino do estado } \\
\text { de São Paulo } \\
\text { Método: Análise Documen- } \\
\text { tal }\end{array}$ & $\begin{array}{l}\text { A SC é uma perspectiva margina- } \\
\text { lizada nos cursos; está isolada em } \\
\text { disciplinas com pequena carga ho- } \\
\text { rária e enormes dificuldades em } \\
\text { estabelecer contatos com a prática } \\
\text { profissional. As disciplinas especí- } \\
\text { ficas, em ambas as faculdades, pa- } \\
\text { recem não ter densidade suficiente } \\
\text { para produzir superações curricu- } \\
\text { lares que permitam o desenvolvi- } \\
\text { mento da própria SC. }\end{array}$ & $\begin{array}{l}\text { São Paulo / } \\
\text { USP e Uni- } \\
\text { camp }\end{array}$ \\
\hline $\begin{array}{c}\text { Santiago, } \\
\text { Pedrosa } \\
\text { e Ferraz } \\
(2016)\end{array}$ & $\begin{array}{l}\text { Analisar no projeto peda- } \\
\text { gógico do curso de EF, bem } \\
\text { como nas DCN dessa área, } \\
\text { o processo formativo liga- } \\
\text { do à atuação em saúde e } \\
\text { ao sistema único de saúde. } \\
\text { Método: Análise Documen- } \\
\text { tal }\end{array}$ & $\begin{array}{l}\text { As disciplinas específicas parecem } \\
\text { não ter densidade suficiente para } \\
\text { produzir superações do enfoque } \\
\text { teórico-metodológico hegemônico } \\
\text { de saúde para um enfoque amplia- } \\
\text { do, em consonância com as neces- } \\
\text { sidades do sistema único de saúde. } \\
\text { Não foi identificada sequer uma } \\
\text { disciplina relacionada à discussão } \\
\text { da SC, o que no citado modelo de } \\
\text { formação seria condição mínima, } \\
\text { considerando as competências } \\
\text { previstas para o futuro profissional } \\
\text { de EF, de acordo com as DCN e pro- } \\
\text { jeto pedagógico do curso. }\end{array}$ & Piauí / UFPI \\
\hline
\end{tabular}

Do ponto de vista metodológico predominou a pesquisa documental, com cinco estudos dedicados à análise de documentos que respaldam os currículos dos cursos de EF das IES investigadas. Os documentos elencados para análise em cada um dos artigos foram: a grade curricular disponível no site oficial das IES atualizada e de domínio público (ANJOS; DUARTE, 2009); Projeto Político Pedagógico, programas das disciplinas e busca por disciplinas com o tema saúde no título, e ementa (BRUGNEROTTO; SIMÕES, 2009); grades das disciplinas obrigatórias em que buscou-se os termos "Saúde Pública", "Saúde Coletiva", "Saúde da Família", "Atenção Básica", "SUS", "Atenção à Saúde", "Integralidade", "Promoção da Saúde", "Educação em Saúde" ou termos similares (COSTA et al., 2012); as ementas de três disciplinas que desenvolviam conteúdos de SC/SP (PASQUIM, 2010); Projeto Pedagógico do Curso e as DCN, por meio das técnicas de leitura analítica e interpretativa, buscan- 
Saúde coletiva e saúde pública no currículo dos cursos de educação física...

Vítor Tavares da Silva • Cintia Ramos Nicoes • Alan Goularte Knuth

do palavras-chave acerca das ideias centrais e secundárias dos documentos (SANTIAGO, PEDROSA, FERRAZ, 2016).

Com isso, nota-se que as cinco investigações aconteceram de modo indireto, destacando algumas limitações ao reforçar a tendência de análise constrangida à presença da palavra "saúde", sendo que essa é uma temática assumidamente transversal nos mais diversos componentes curriculares da formação em EF. Dessa maneira, torna-se importante ressaltar o papel de outras abordagens e procedimentos metodológicos que se aproximem desta transversalidade, como observações, entrevistas, entre outros.

Um exemplo se destaca no prisma da tríade universitária, em que, por meio da análise documental, os estudos, de forma geral, não contemplam as experiências de formação em projetos de extensão e pesquisa nas IES estudadas. Apenas Pasquim (2010), por meio da técnica de entrevista, permitiu emergir e destacar resultados que atentam à importância da temática da saúde para além do ensino de base documento-curricular, de forma a alcançar a relevância de grupos de pesquisa e projetos de extensão alicerçados nas disciplinas curriculares.

Em sequência, outro investimento metodológico foi o estudo de caso descritivo-exploratório, quando foram realizados dois grupos focais, compostos por PEF com experiência na APS e em fase final do curso (FALCl; BELISARIO, 2013). Em todos os artigos incluídos nesta revisão foram verificados os currículos de ambas as modalidades de ensino, assim, tanto os cursos de bacharelado quanto os de licenciatura fizeram parte das análises.

Dentre os estudos analisados, um investigou a temática da SP e SC nos currículos dos cursos de EF em uma abrangência nacional. Costa et al. (2012) pesquisaram 61 IES das capitais brasileiras a partir de consultas na grade curricular dessas instituições.

Num contexto local, três estudos investigaram cursos de EF de IES públicas e privadas, alcançando estados da região sudeste. Em São Paulo, no estudo de Anjos e Duarte (2009), foram pesquisados os currículos de cinco Instituições (4 públicas e 1 privada). Já 
Saúde coletiva e saúde pública no currículo dos cursos de educação física...

Vítor Tavares da Silva • Cintia Ramos Nicoes • Alan Goularte Knuth

Pasquim (2010), dedicou-se aos cursos de graduação em EF, especialmente na Universidade de São Paulo (USP) e na Universidade Estadual de Campinas (Unicamp). Ainda na região Sudeste, tem-se o estudo de Falci e Belisario (2013), realizado na Universidade Federal de Minas Gerais (UFMG).

Na região Nordeste, Santiago, Pedrosa e Ferraz (2016) analisaram o curso de EF da Universidade Federal do Piauí (UFPI). Por fim, na região Sul figurou o estudo de Brugnerotto e Simões (2009) sobre os currículos de 12 cursos (6 bacharelados e 6 licenciaturas) de 6 IES públicas do Paraná.

Com isso, os seis estudos brasileiros analisaram currículos de cursos de EF com vistas a observar as noções de SP e SC, dentre os quais a pesquisa documental predomina e regionalmente não se destacam pesquisas nas IES do Norte e Centro-oeste do Brasil. Esse contexto corrobora o cenário de investigações científicas nas regiões Norte e Centro-oeste, em que se destacam uma quantidade menor de estudos científicos quando comparadas às demais regiões do país. O que não é diferente quando se trata de estudos envolvendo temáticas sensíveis à EF e à saúde (SIDONE; HADDAD; MENA-CHAL, 2016).

Os resultados dos estudos coincidiram no que se refere à predominância de disciplinas da saúde veiculadas ao ensino do bacharelado. Ainda que denotem insuficiências com demandas da EF em diálogos com o SUS, tal modalidade de formação é a que, hegemonicamente, recebe maiores investimentos disciplinares. Outros aspectos relacionados ao processo formativo também evidenciam abordagens de cunho biomédico e uma noção de saúde desajustada da complexidade das intervenções da EF, elementos que serão ampliados a seguir.

Em IES do estado de São Paulo, os resultados do estudo de Anjos e Duarte (2009) indicam que o perfil de formação de PEF é voltado à atividade física e a prescrever, segundo diagnóstico e avaliação, a partir de protocolos regidos por parâmetros puramente biológicos. Dentre os cursos investigados, os currículos de 
Saúde coletiva e saúde pública no currículo dos cursos de educação física...

Vítor Tavares da Silva • Cintia Ramos Nicoes • Alan Goularte Knuth

bacharelado têm mais respaldo em saúde nesta perspectiva quando comparado com a licenciatura; no primeiro caso predominam disciplinas de abordagem curativa e prescritiva. Por fim, nenhuma das instituições possui disciplinas dedicadas à SC e/ou à SP, de maneira que também não são articulados estágios no serviço público de saúde (ANJOS; DUARTE, 2009).

Ainda em São Paulo, em um estudo envolvendo a Universidade de São Paulo (USP) e a Universidade Estadual de Campinas (Unicamp), Pasquim (2010) verificou o ensino de SC nos cursos de graduação em EF. A pesquisa destaca que os currículos de ambas IES, além de muito distintos, parecem não ter profundidade suficiente para oferecer uma formação densa em SC.

Já Brugnerotto e Simões (2009), ao analisar os currículos de 12 cursos ( 6 bacharelados e 6 licenciaturas) de seis IES públicas do Paraná, identificaram componentes curriculares sensíveis à temática da SC e da SP em todos os cursos de bacharelado, porém em apenas três dos cursos de licenciatura e com diferentes enfoques: humanista para a licenciatura e biológico para o bacharelado.

Além disso, o estudo mostra a necessidade de amadurecimento das propostas encontradas nos currículos, principalmente no modo pelo qual eles são implantados. Os autores enquadram os currículos em uma tendência técnico-linear, que pressupõe uma área já existente no "mercado". Desse modo, necessita de recursos humanos para dar suporte aos objetivos provenientes da sua existência e vem se mostrando ineficiente enquanto mecanismo de formação profissional dentro de seus próprios ideais.

Nesse sentido, Falci e Belisario (2013), ao investigarem a formação do PEF na UFMG para sua inserção na APS, também sugerem que a formação em graduação necessita de uma reestruturação curricular. Essa indução minimizaria as limitações apresentadas diante do desenvolvimento da temática da SC e da SP no curso de EF da universidade. O curso de EF na UFMG foi avaliado por egressos com uma formação frágil para a atuação na APS. Os autores do estudo apontam que devem ser fomentadas mais oportunidades 
Saúde coletiva e saúde pública no currículo dos cursos de educação física... Vítor Tavares da Silva • Cintia Ramos Nicoes • Alan Goularte Knuth

de aproximação da realidade profissional com a SP por meio de estágios e a transversalidade do tema na grade curricular.

Ainda nesse contexto, Santiago, Pedrosa e Ferraz (2016), ao analisarem o projeto pedagógico do curso do curso de EF da UFPI, acerca do processo formativo ligado à atuação em saúde e no SUS, identificaram inúmeros elementos na formação que subsidiariam uma melhor atuação em saúde. Em destaque, principalmente, a não observação de disciplinas ou espaços para a apropriação desse debate. Dentre as 41 disciplinas dos cursos, apenas a disciplina "Higiene, Saúde e Meio Ambiente" apresentou o conteúdo SC em sua ementa, o qual não se traduziu no plano de disciplina. Segundo o estudo, o currículo não assume potência teórica suficiente para produzir superações do enfoque teórico-metodológico hegemônico de saúde para um enfoque ampliado expresso nos mais recentes avanços dos estudos em SC e SP.

Como estratégia de análise, apresentamos a seguir alguns trabalhos não captados pela revisão sistemática, mas que aportam contribuições analíticas ao tema.

Luiz (2016), em tese que objetivou investigar as disciplinas componentes da matriz curricular dos cursos de graduação em EF e relação com a saúde, identificou que as disciplinas do agrupamento "Saúde Pública e Coletiva" corresponderam a apenas 18,0\% do total. Apesar da existência de disciplinas ligadas às Ciências Humanas e Sociais na formação básica com enfoque em saúde, esses conteúdos parecem ainda muito desvalorizados pelo caráter eletivo dentro do currículo. Em suma, apesar dos avanços, os conhecimentos teóricos e práticos do campo da SC ainda não estão consolidados nos currículos, evidenciando que a EF ainda está em processo de afirmação enquanto profissão da saúde no contexto da SP (SILVA, 2016).

Nessa ênfase, ao analisar a fala dos discentes dos cursos de EF da Universidade Federal do Rio Grande do Sul (UFRGS) apresentada nos relatórios de avaliação dos currículos, Dahlke (2017) destaca uma resistência em relação à superação do paradigma bio- 
Saúde coletiva e saúde pública no currículo dos cursos de educação física...

Vítor Tavares da Silva • Cintia Ramos Nicoes • Alan Goularte Knuth

médico na EF, quando os alunos marcam posições que entendem as disciplinas biomédicas como essenciais, bem como a exaltação dos conhecimentos técnicos e científicos e as disciplinas socioculturais como desnecessárias.

Silva (2016), ao buscar compreender a preparação profissional para atuação na SC de duas graduações de EF, com ênfase de formação para a área da saúde, conclui que estas visões antagônicas de práticas à saúde emergiram em um contexto de inconsistências e incoerências, apontando que a formação em EF ainda não tem definido o conceito de saúde subjacente à sua prática profissional, especificamente para o âmbito da SC.

Como destacam Fraga, Carvalho e Gomes (2012), os princípios do SUS ainda não encontram eco na atuação acadêmica e profissional na EF. Luiz (2016) também evidencia um descompasso entre a formação e as políticas públicas que balizam a EF nas equipes multiprofissionais e serviços de saúde, identificando que um debate em relação à reorientação da formação está ainda sendo inaugurado na EF. A APS, por exemplo, onde se destacam o maior número de PEF, ainda se constitui como um espaço desafiador de intervenção para o profissional de EF, pois os saberes teóricos e práticos necessários para atuação neste setor ainda não estão consolidados na preparação profissional (SILVA, 2016).

A partir do exposto, mostra-se um cenário de incertezas em relação à SC e à SP na EF, considerando que a graduação é um dos mais importantes fatores a serem analisados na busca por uma melhor atuação no contexto da SC. Dessa maneira, ao ser considerada como profissão da saúde, a EF necessita ter sua formação alinhada ao SUS (LOCH et al., 2011), e a SP demanda pautar/ordenar a formação dos recursos humanos na área, como previsto na constituição brasileira (BRASIL, 1988).

Portanto, entende-se que o advocacy pelas políticas públicas deveria perpassar os currículos da formação em EF, e nisso inclui-se a saúde, bem como aquelas que tematizam o esporte, a cultura, a assistência social e o lazer. Recentemente, o conteúdo apre- 
Saúde coletiva e saúde pública no currículo dos cursos de educação física...

Vítor Tavares da Silva • Cintia Ramos Nicoes • Alan Goularte Knuth

sentado pela Portaria № 2.979, de 12 de novembro de 2019, que versa sobre o novo modelo de financiamento de custeio da APS no âmbito do SUS (BRASIL, 2019), pode representar ainda mais ameaças para a EF vinculada à SC e SP, e, com isso, emerge mais um debate pertinente aos estudos acerca formação profissional em $\mathrm{EF}$, bem como as próprias práticas curriculares.

Diante disso, não se pode assumir uma posição maniqueísta em que a saúde inundaria os currículos de EF, mas sim de entender sua limitada presença até o momento. Outro aspecto que emerge nos estudos aqui revisados é a necessidade de uma reformulação curricular nos cursos superiores em EF. Entretanto, para discutir mudanças para a formação em EF no campo da saúde, é necessário entender, preliminarmente, que o campo da formação profissional - em qualquer área do conhecimento - é um território de dilemas, conflitos e tensões.

Destaca-se, assim, que o debate sobre o SUS é fundamental na formação acadêmica, devendo-se discutir e vivenciar uma prática profissional pautada em seus princípios (ROCHA; CENTURIÃO, 2007). É necessário considerar referenciais teóricos que alicercem o entendimento sobre a saúde também em sua base biopsicossocial, considerando os determinantes da saúde. Como apontam Falci e Belisario (2013), as maiores oportunidades de aproximação da graduação com a realidade da SP se dão por meio de estágios, da transversalidade do tema na grade curricular e da interação com outras áreas do conhecimento através de disciplinas integradas.

Nessa perspectiva, pautar a SC e, por conseguinte, o referencial das Ciências Humanas e Sociais como fundamento, significa compreender a EF e a própria saúde, dando legitimidade para a vida e os múltiplos e complexos determinantes das ações humanas. Portanto, implica perceber as influências culturais, condições de vida e as distintas possibilidades de acesso a bens e serviços como condicionantes do modo de viver e, ainda, compreender o ser humano socialmente, sem segmentá-lo, reduzi-lo ou mesmo universalizá-lo (FREITAS; BRASIL; SILVA, 2006). 
Saúde coletiva e saúde pública no currículo dos cursos de educação física...

Vítor Tavares da Silva • Cintia Ramos Nicoes • Alan Goularte Knuth

Para isso, a discussão pode ser facilmente direcionada à ênfase das concepções de currículo assumidas nos estudos aqui analisados. Porém, os estudos não se debruçam centralmente em demarcar mais fortemente as concepções curriculares das IES investigadas, principalmente em detrimento de perseguir as concepções do tema da saúde, utilizando o currículo como uma fonte e meio de discutir seus achados na ênfase da SP e da SC e dos enfoques adotados nos cursos. Quem mais se aproxima dessa demanda são Brugnerotto e Simões (2009), ao destacarem o currículo como um instrumento de "poder" que representa os interesses de classes. Os autores alertam para o fato de que, na lógica política, a classe dominante, que tem no estado a sua instância de poder, direciona as diretrizes e reformas curriculares de forma vertical e afastada de princípios democráticos.

Já na perspectiva dos perfis de formação em ambas as modalidades, em relação ao tema das políticas públicas de saúde brasileiras, emergem vários diálogos também no campo da Educação, principalmente ao sinalizar para os conceitos de educação em saúde, educação permanente em saúde, humanização, integralidade, interprofissionalidade. Entretanto, essa ênfase assume maior destaque e aprofundamento apenas nos resultados do estudo de Brugnerotto e Simões (2009), em que tais conhecimentos se afastam da formação em bacharelado, assumindo maior destaque nas licenciaturas.

Considerado o exposto, no que tange a um dos aspectos basais da discussão em torno da temática curricular nos cursos de graduação em EF, emergem as DCN. A reforma curricular do ano de 2004 se consolidou como a mais importante transformação nos processos formativos da EF evidenciados nesta revisão, criando um modelo hegemônico dentro do contexto do ensino superior nacional em EF. Dessa maneira, a adesão à reforma nos cursos avaliados se mostrou como a última grande reestruturação curricular, destacando uma das questões mais discutidas quanto à SC e à SP nos currículos: a divisão entre licenciatura e bacharelado, e a EF enquanto profissão da saúde. 
Saúde coletiva e saúde pública no currículo dos cursos de educação física...

Vítor Tavares da Silva • Cintia Ramos Nicoes • Alan Goularte Knuth

Segundo Taffarel (2012), as DCN geraram uma fragmentação do conhecimento e lacunas na formação, principalmente pela divisão e negação do conhecimento e postos de trabalho. Nesse sentido, Pasquim (2010), ao usar a expressão "distúrbio bipolar institucional", destacou que a fragmentação da formação em dois polos, muitas vezes entendidos como antagônicos - do licenciado e sua visão restrita à escola, e do bacharel e sua visão restrita ao treinamento -, desfavorece uma prática integral na EF e as aproximações com campos de conhecimentos como a SC.

No destaque da SP, Fraga, Carvalho e Gomes (2012) alertam que o SUS não é sequer mencionado nas DCN, além de ser um fato que coincide com uma falta de compreensão das demandas do SUS para a EF. A menção mais clara acerca da temática da saúde nas diretrizes se destaca ao ponderarem afirmações que sinalizam competências profissionais na EF relacionadas à saúde - responsável pela prevenção, promoção, proteção e reabilitação da saúde (BRASIL, 2004).

Portanto, consolida-se um cenário preocupante, pois as investigações analisadas nesta revisão destacam a ausência/desinvestimento de disciplinas e conteúdos relacionados à SC e à SP. Dentre as escassas experiências de disciplinas de saúde evidenciadas nos estudos mais restritos geograficamente, os conteúdos foram avaliados como insuficientes para o desenvolvimento de competências para a atuação nos diferentes níveis de atenção da SP.

Diante disso, recentemente, no ano de 2018, por meio da resolução $n^{\circ} 6$ de 18 de dezembro das Diretrizes Curriculares Nacionais dos Cursos de Graduação em EF, foi instituído que o curso terá ingresso único, destinado tanto ao bacharelado quanto à licenciatura, porém mantendo a divisão na formação durante o decorrer do curso. Para esse processo de reestruturação, as IES terão até o ano de 2021 para se adequarem (BRASIL, 2018).

Essas diretrizes, questionadas por alguns segmentos da EF em razão da reduzida interlocução com as IES, comportam um olhar maior para os temas de SC e SP e, provavelmente, produzirão mo- 
Saúde coletiva e saúde pública no currículo dos cursos de educação física...

Vítor Tavares da Silva • Cintia Ramos Nicoes • Alan Goularte Knuth

vimentos e efeitos curriculares nos próximos anos, bem como resistências e rupturas. Com isso, o interesse acadêmico-científico se faz necessário também na ênfase de como o cenário desenhado nos últimos 15 anos acerca da SC e da SP no currículo da EF pode ser reconfigurado.

\section{Conclusão}

Por meio desta revisão foi possível destacar uma escassa produção de artigos originais interessada nos temas SP e SC nos currículos de EF das IES no Brasil. Diante disso, a análise dos estudos revisados permite concluir, centralmente, que as temáticas da SC e da SP não assumem grande expressão nos currículos da EF. Essas temáticas aparecem majoritariamente na formação de cursos de bacharelado, de maneira que, mesmo presentes em uma minoria de currículos, figuram de maneira frágil em relação à sua abordagem, com insuficiente densidade para as interlocuções com o campo de conhecimento e prática da SC e os serviços públicos de saúde.

Vale a pena destacar que as mudanças curriculares recentemente anunciadas, que incorporam objetivamente os temas da SP e da SC, produzirão mudanças em direção a contemplar seus conhecimentos e práticas, sem que necessariamente se rompa com a abordagem superficial, individualista e biológica de saúde, que, aparentemente, predomina quando os discursos e práticas se valem do tema saúde em termos de formação profissional no Brasil.

Contudo, sabidamente, o processo de formação nos cursos de EF no Brasil é maior, mais complexo e alcança outros temas, para além de sua relação com EF e saúde discutida e analisada nesta revisão. Dessa forma, emerge o alerta para que mais estudos sejam desenvolvidos neste enfoque, permitindo que maiores compressões sejam fomentadas e transcendam a realidade da formação superior em EF, reconhecendo suas potencialidades e contradições históricas em relação ao campo da saúde. 
Saúde coletiva e saúde pública no currículo dos cursos de educação física... Vítor Tavares da Silva • Cintia Ramos Nicoes • Alan Goularte Knuth

\section{Referências}

ANJOS, Tatiana Coletto dos; DUARTE, Ana Cláudia Garcia de Oliveira. A Educação Física e a estratégia de saúde da família: formação e atuação profissional. Physis, Rio de Janeiro, v. 19, n. 4, p. 1127-1144, 2009. Disponível em: http://www.scielo.br/scielo.php?script=sci_arttext\&pi$\mathrm{d}=$ =S0103-73312009000400012\&lng=en\&nrm=iso. Acesso em: 14 jan. 2019.

BRASIL. Conselho Nacional de Educação. Câmara de Educação Superior. Resolução n 07/2004, de 18 de fevereiro de 2004. Institui as Diretrizes Curriculares Nacionais para os cursos de graduação em Educação Física, em nível superior de graduação plena. Brasília, 2004. Disponível em: http://portal.mec.gov.br/cne/ arquivos/pdf/2007/res07_04.pdf. Acesso em: 12 nov. 2018.

\section{BRASIL. Constituição da República Federativa do Brasil de}

1988. Disponível em: http://www.planalto.gov.br/ccivil_03/constituicao/constituicao.htm. Acesso em 03 out. 2018.

BRASIL. Ministério da Saúde. Gabinete do Ministro. Portaria $\mathrm{n}^{\circ}$ 2.979, de 12 de novembro de 2019. Institui o Programa Previne Brasil, que estabelece novo modelo de financiamento de custeio da Atenção Primária à Saúde no âmbito do Sistema Único de Saúde, por meio da alteração da Portaria de Consolidação $n^{\circ}$ 6/GM/MS, de 28 de setembro de 2017. Disponível em: http:// www.in.gov.br/web/dou/-/portaria-n-2.979-de-12-de-novembro-de-2019-227652180. Acesso em: 10 dez. 2019.

BRUGNEROTTO, Fábio; SIMOES, Regina. Caracterização dos currículos de formação profissional em Educação Física: um enfoque sobre saúde. Physis, Rio de Janeiro, v. 19, n. 1, p. 149-172, 2009. Disponível em: http://www.scielo.br/scielo.php?script=sci_arttext\&pid=S0103-73312009000100008\&lng=en\&nrm=iso. Acesso em: 10 jan. 2019. 
Saúde coletiva e saúde pública no currículo dos cursos de educação física...

Vítor Tavares da Silva • Cintia Ramos Nicoes • Alan Goularte Knuth

CARVALHO, Fabio Fortunato Brasil de; NOGUEIRA, Júlia Aparecida Devidé. Práticas corporais e atividades físicas na perspectiva da Promoção da Saúde na Atenção Básica. Ciênc. saúde coletiva, Rio de Janeiro, v. 21, n. 6, p. 1829-1838, 2016. Disponível em: http://www.scielo.br/scielo.php?script=sci_arttext\&pi$\mathrm{d}=\mathrm{S} 1413-81232016000601829 \& \mathrm{lng}=\mathrm{en} \& \mathrm{nrm}=\mathrm{iso}$. Acesso em: 10 jan. 2019.CARVALHO, Yara Maria de. Educação Física e Saúde Coletiva: uma introdução. In: LUZ, M.T. Novos saberes e práticas em Saúde Coletiva: estudo sobre racionalidades médica e atividades corporais. $3^{a}$ ed. São Paulo: Hucitec, 2007. p. 19-34.

CARVALHO, Yara Maria de; CECCIM, Ricardo Burg. Formação e educação em saúde: aprendizados com a Saúde Coletiva. In: CAMPOS, Gastao Wagner De Sousa. et al. (Org.). Tratado de Saúde Coletiva. São Paulo: Hucitec, 2006. p.149-182.

COLL, Cesar. Psicologia e Currículo: uma aproximação psicopedagógica à elaboração do currículo escolar. São Paulo: Ática, 2002.

COSTA, Larissa Chaves et al. Formação profissional e produtividade em saúde coletiva do Profissional de Educação Física. Revista Brasileira de Atividade Física \& Saúde, v. 17, n. 2, p. 107-113, 2012. Disponível em: http://rbafs.org.br/RBAFS/article/view/1226. Acesso em: 20 jan. 2019.

\section{DAHLKE, Ana Paula. A saúde nos currículos de formação}

superior em educação física: uma análise do discurso de estudantes dos cursos de licenciatura e bacharelado da UFRGS. 165 f. Dissertação (Mestrado) - Universidade Federal do Rio Grande do Sul, Escola de Educação Física, Programa de Pós-Graduação em Ciências do Movimento Humano, Porto Alegre, BR-RS, 2017. Disponível em: https://www.lume.ufrgs.br/handle/10183/170225. 20 jan. 2019.

FALCI, Denise Mourão; BELISARIO, Soraya Almeida. A inserção do profissional de educação física na atenção primária à saúde e os desafios em sua formação. Interface (Botucatu), Botucatu, v. 17, n. 47, p. 885-899, 2013. Disponível 
Saúde coletiva e saúde pública no currículo dos cursos de educação física...

Vítor Tavares da Silva • Cintia Ramos Nicoes • Alan Goularte Knuth

em: http://www.scielo.br/scielo.php?script=sci_arttext\&pi$d=S 1414-32832013000400010 \& \mid n g=e n \& n r m=i s o$. Acesso em: 12 jan. 2019.

FRAGA, Alex Branco; CARVALHO, Yara Maria de; GOMES, Ivan Marcelo. Políticas de formação em educação física e saúde coletiva. Trab. educ. saúde, Rio de Janeiro, v. 10, n. 3, p. 367-386, 2012. Disponível em: http://www.scielo.br/scielo.php?script=sci_arttext\&pid=S1981-77462012000300002\&lng=en\&nrm=iso. Acesso em: 20 jan. 2019.

FREITAS, Fabiana Fernandes de; BRASIL, Fernanda Kundrát; SILVA, Cinthia Lopes da. Práticas corporais e saúde: novos olhares.

Revista Brasileira de Ciências do Esporte, Campinas, n. 3, v. 27, p. 169-183, 2006. Disponível em: http://www.rbce.cbce.org.br/ index.php/RBCE/article/view/81/87. Acesso em: 19 jan. 2019.

LOCH, Mathias Roberto et al. A Saúde Pública nos anais do Congresso Brasileiro de Atividade Física e Saúde (1997-2009): revisão sistemática. Revista Brasileira de Atividade Física \& Saúde, v. 16, n. 2, p. 162-167, 2011. Disponível em: http://rbafs. org.br/RBAFS/article/view/577/585. Acesso em: 15 fev. 2019.

LUIZ, Angela Rodrigues. Ensino sobre saúde pública e coletiva nos cursos de graduação em educação física no Brasil. 2016. 89 f. Tese (Doutorado em Ciências da Saúde) - Universidade Federal de Goiás, Goiânia, 2016. Disponível em: https://repositorio.bc.ufg.br/tede/bitstream/tede/5670/5/Tese\%20-\%20 Angela\%20Rodrigues\%20Luiz\%20-\%202016.pdf. Acesso em: 16 fev. 2019.

PAIM, Jairnilson Silva. Sistema Único de Saúde (SUS) aos 30 anos. Ciênc. saúde coletiva, Rio de Janeiro, v. 23, n. 6, p. 1723-1728, 2018. Disponível em http://www.scielo.br/scielo.php?script=sci_arttext\&pid=S1413-81232018000601723\&lng=en\&nrm=iso. Acesso em: 12 jan. 2019.

PASQUIM, Heitor Martins. A saúde coletiva nos cursos de graduação em Educação Física. Saude soc., São Paulo, v. 19, n. 1, p. 193-200, 2010. Disponível em: http://www.scielo.br/scielo.php?s- 
Saúde coletiva e saúde pública no currículo dos cursos de educação física...

Vítor Tavares da Silva • Cintia Ramos Nicoes • Alan Goularte Knuth

cript=sci_arttext\&pid=S0104=12902010000100016-\&lng=en\&nrmiso. Acesso em: 20 jan. 2019.

ROCHA, Vera Maria; CENTURIÃO, Carla Haas. Profissionais da saúde: formação, competência e responsabilidade social. In: FRAGA, Alex Branco; WACHS, Felipe (Org.). Educação Física e saúde coletiva: políticas de formação e perspectivas de intervenção. Porto Alegre: Editora da UFRGS, 2007. p. 17-32.

SANTIAGO, Maria Luci Esteves; PEDROSA, José Ivo Santos; FERRAZ, Alex Soares Marreiros. A formação em saúde à luz do projeto pedagógico e das diretrizes curriculares da educação física. Movimento, Porto Alegre, p. 443-458, 2015. Disponível em: https://seer.ufrgs.br/Movimento/article/view/57988. Acesso em: 29 fev. 2019.

SIDONE, Otávio José Guerci; HADDAD, Eduardo Amaral; MENACHALCO, Jesús Pascual. A ciência nas regiões brasileiras: evolução da produção e das redes de colaboração científica. Transinformação, Campinas, v. 28, n. 1, p. 15-32, 2016. Disponível em: http://www.scielo.br/scielo.php?script=sci_arttext\&pid=S0103-37862016000100015\&Ing=en\&nrm=iso. Acesso em: 29 fev. 2019.

SILVA, Tomaz Tadeu da. Documentos de Identidade: uma introdução às teorias do currículo. Belo Horizonte: Autêntica, 2005.

SILVA, Luiz Henrique. A formação em educação física para atuação na saúde. 322 f. Tese (doutorado) - Universidade Estadual Paulista, Instituto de Biociências de Rio Claro, 2016. Disponível em: https://repositorio.unesp.br/bitstream/handle/11449/139515/silva_Ih_dr_rcla.pdf?sequence=6\&isAllowed=y. Acesso em 12 jan. 2019.

TAFFAREL, Celi Zulke. Formação de professores de educação física: diretrizes para a formação unificada.. Kinesis, [s.l.], 2012. Disponível em: https://periodicos.ufsm.br/kinesis/article/ view/5726. Acesso em: 20 jan. 2019. 
Saúde coletiva e saúde pública no currículo dos cursos de educação física...

Vítor Tavares da Silva · Cintia Ramos Nicoes • Alan Goularte Knuth

\section{Financiamento}

Esse trabalho contou com apoio e financiamento da Coordenação de Aperfeiçoamento de Pessoal de Nível Superior Brasil (CAPES).

\section{Publisher}

Universidade Federal de Goiás. Faculdade de Educação Física e Dança. Publicação no Portal de Periódicos UFG. As ideias expressadas neste artigo são de responsabilidade de seus autores, não representando, necessariamente, a opinião dos editores ou da universidade. 